\title{
FIRST RESULTS OF THE DUO PROGRAM
}

\author{
C. ALARD \\ Centre d'Analyse des Images de l'INSU, \\ Batiment Perrault, Observatoire de Paris, \\ 61 Avenue de l'Observatoire, \\ F-75014, Paris, France
}

\begin{abstract}
The DUO (Disk Unseen Objects) program is a project with the main goal of searching for galactic dark matter in a field towards the Galactic Bulge (Alard et al. 1995b). In this paper I present the results obtained from the analysis of half of the data collected during the 1994 season. I start with a brief description of the DUO project, and I will continue with an analysis of the microlensing candidates that I found. In all I found 13 microlensing events in the DUO data, including a double lens event. A model of the double lens light curve predicts a blending effect. The presence of the blended component was confirmed by a gravity center shift during the event. Taking into account the model prediction and the shift, we could predict the exact geometry of the blend. This prediction was recently confirmed by direct imaging of the blend under good seeing conditions. The durations of the single lens events are quite short, but the durations can be seriously affected by blending. I propose a simple experimental test to quantify this duration bias. In the conclusion I emphasize the great importance of a good knowledge of the inner Galactic structure to get a reliable quantitative description of microlensing towards the bulge. Finally I will show that the large number of variables stars found in the data could be a powerful tool to probe this structure.
\end{abstract}

\section{Introduction}

The data presented here were collected during the 1994 observing season with the ESO $1 \mathrm{~m}$ Schmidt telescope. The season lasted from April 5 to October 10. Our sampling contains some gaps due to moon periods and to 
poor weather conditions, especially at the beginning of the season.

The Schmidt telescope provides photographic plates of $28 \mathrm{~cm} \times 28 \mathrm{~cm}$. The scale is $67^{\prime \prime} / \mathrm{mm}$, giving a wide field of $5.2 \times 5.2$ degrees. Two B plates (IIIaJ) and one R plate (IIIaF) were taken during each photometric night. The mean time interval between two $B$ plates was 1.5 hours, with the $\mathrm{R}$ plate taken just in between.

\subsection{PLATE SCANNING}

All the plates were scanned with the MAMA machine in Paris Observatory. Mama (Machine Automatique a Mesurer pour l'Astronomie, developed and operated by INSU/CNRS) is a high speed microdensimeter providing images with a pixel of $10 \mu \mathrm{m}$. All the images were reduced with the standard package of the DUO project (Alard 1995).

\subsection{THE LIMITING MAGNITUDES}

The bulge stars that can act as sources for microlensing have two different locations in a typical color magnitude diagram: the giant clump, and the region of the bulge turn-off. The DUO field is rather far from the Galactic Center, so the giant clump is not very populated; as a result, most of the bulge stars will come from the turn-off region. In the B band, the limit is just faint enough to reach the turn-off proper, but in $R$ the limit is a few hundredths of a magnitude brighter. This implies that most of the events are found in the $\mathrm{B}$ band, and quite close to the limit; in R most of these events are a little bit too faint to provide reliable light curves, especially if the star is badly blended. By chance the double lens event was well separated from its neighbors on the plate and it had a large amplitude, which leads to a rather nice light curve in $R$. The other events are all blended and very faint on the $\mathrm{R}$ plates. Thus, the main problem is that for such very faint fluxes the response of the plate is almost null and highly non-linear. In addition, the response of the plate is seriously affected if a brighter companion lies within a few pixels from the event. It makes the extracted signal very noisy and almost impossible to calibrate.

\section{The Microlensing candidates}

\subsection{THE SELECTION OF THE CANDIDATES}

The candidates were selected using a method similar to the one adopted by OGLE (Udalsky et al. 1994). However the definition of the baseline value had to be changed, because OGLE uses the measurements taken in the other season to estimate the baseline, while DUO has only 1 season 


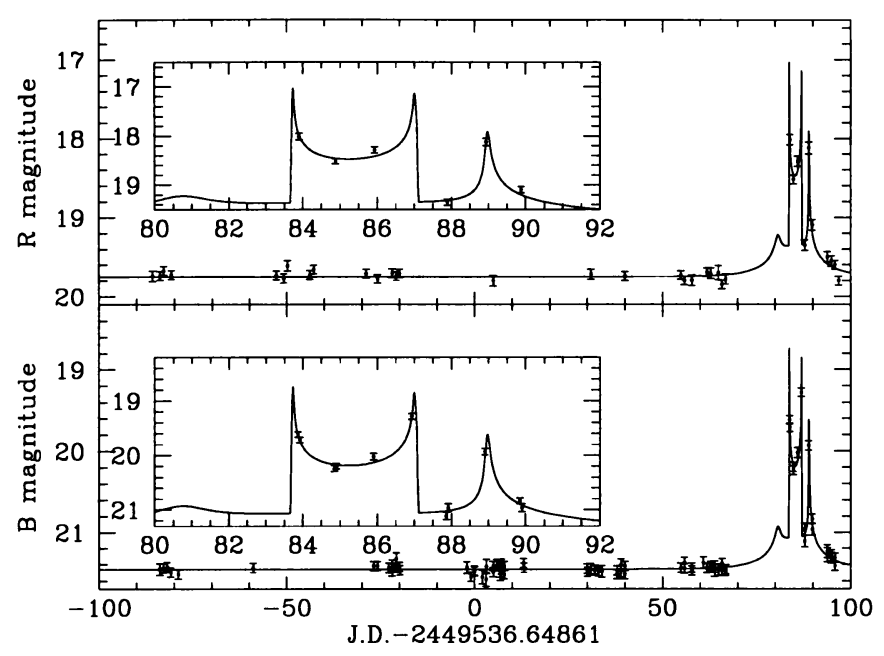

Figure 1. Light curve of DUO2

of observations. I defined the baseline value as the minimum average of 5 consecutive points all over the light curve. All curves with more than 4 consecutive deviating points over 3 sigmas were selected, and fitted to the theoretical light curve. In the last step, a theoretical microlensing light curve was fitted to the data, and the standard rms deviation $\mathrm{r} 1$ calculated. The $\mathrm{rms} \mathrm{r} 2$ to the fit of a constant line was also computed. The final criterion was that the ratio $\mathrm{r} 2 / \mathrm{r} 1$ should be greater than 2 .

\subsection{A DOUBLE LENS}

One of the microlensing candidates discovered, DUO 2, shows very unusual variability. The star remained stable for more than 150 days before it brightened by more than two magnitudes in 6 days in both the $B$ and $R$ bands. The light curves are achromatic during the variability (Figure 1).

A binary lens model was fitted to the observations (Alard et al. 1995a). The masses of the lenses are quite small, with the companion possibly in the range of a brown dwarf or even a few times of Jupiter. Our first model did not include any blending, and one point gave a 4 sigma deviation in both the $\mathrm{B}$ and $\mathrm{R}$ bands. After adding a blending parameter we had a very good fit to the data, although with about 10 data points during the event we can wonder about the significance of this result. But we found other strong evidence for blending: a gravity center shift was observed during the event at a 4 sigma confidence level. This simply means that the gravity center is shifted towards the amplified component during the event. The 


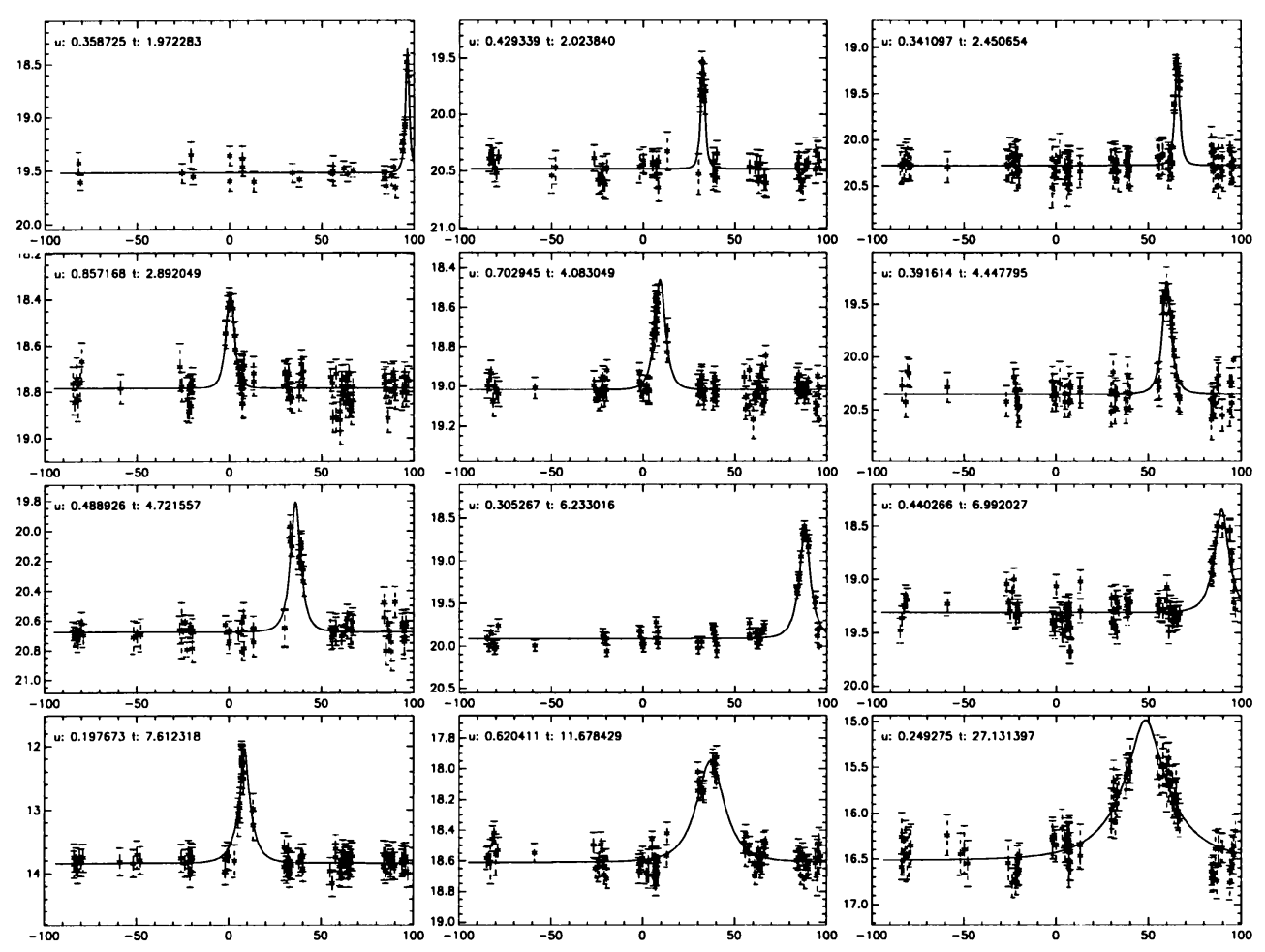

Figure 2. Light curves of the 12 single lens events in the B band (the $\mathrm{x}$ axis is graduated in days)

model provides the flux ratio of the components which, combined with the shift in both coordinates, allows us calculate all the blend parameters: the separation, the angle between the components, and their flux ratio.

This blend model was recently tested using two CCD images taken under good seeing conditions (Van Der Hooft 1995; Szymański \& Udalski 1995). Both images show two components. Their separation, position angle, and flux ratio are in good agreement with our model.

\subsection{THE SINGLE LENS EVENTS}

Twelve candidates satisfied all the cuts described in $\S 2.1$. In figure 2 we can see their light curves in the B band. The events are in order of increasing durations. Many of them have quite short durations, in the range 1.8 days to 5 days, which could be consistent with lenses like low mass stars or brown dwarfs. However, the duration can be seriously affected by blending, and high resolution images are needed before reaching any definitive conclusions about the lens characteristics of these events. 


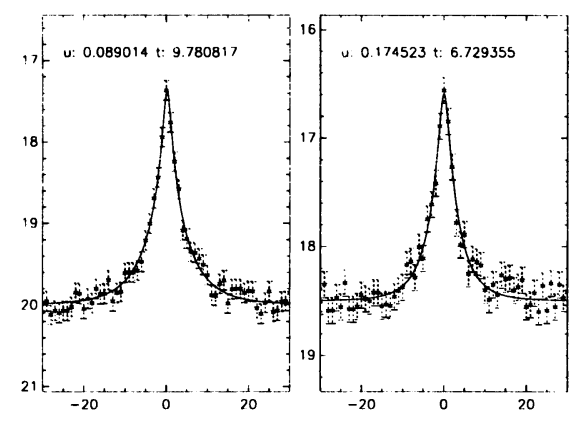

Figure 3. Two simulated light curves with a duration of 10 days: (left) an unblended light curve, and (right) the star blended with a same magnitude companion. The impact parameter $u$, and the duration $t$, of the unblended fitted light curve are indicated. Notice the bias in the estimated duration of the right curve.

\subsection{BIAS IN THE EVENT DURATIONS}

In most cases a theoretical unblended light curve can be fitted to a blended event with a good agreement, as illustrated in figure 3 . But we notice that if the fit is consistent with the errors of a typical light curve event like in figure 3 , the impact parameter $\mathrm{u}$, and the duration $\mathrm{t}$, are seriously affected. The event durations strongly decrease with the blending effect. This effect would induce an overestimate of the lens mass.

A possible experimental test: Among the two kinds of possible sources for microlensing, one is quite bright: the clump giants. These stars are brighter than most of the other stars in a bulge field, so the effect of blending on the parameter estimate should be negligible. Following this idea, it is quite straight forward to compare the duration of events for these clump stars to the duration of events for the fainter turn-off stars. We should find that the mean duration of the events for clump stars is longer than for the turn off stars. Unfortunately there are only 2 clump events for DUO, but it would be probably rather simple to check this effect in the MACHO experiment.

\section{The Inner Galactic Structure}

The first step before any serious modeling of microlensing towards the bulge is to determine the structure of the inner Galaxy. At the present time the existence of a bar near the center of our galaxy is well established (Weinberg 1992), but the parameters of the bar model are still poorly known. The inclination of the bar and its size have a very significant effect on the microlensing rate. But some questions about the structure of the disk are also of great importance: does the density of the disk drop at a few kilopar- 

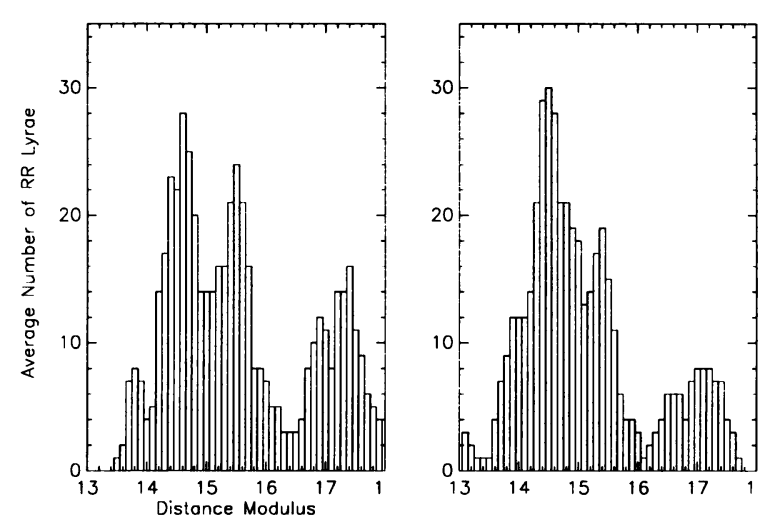

Figure 4. RR lyrae histograms of 2 selected regions (see text for explanations)

sec from the center (Paczyński et al. 1994)? The large number of variable stars found in the data could seriously help answer these questions. I illustrate this idea by showing some histograms of the RR Lyrae variable distribution. Figure 4 presents histograms of two selected regions with low and homogeneous extinction. The bulge is visible as the peak at a distance modulus of $14.5(8 \mathrm{Kpc})$, but a strong peak at $15.5(12.5 \mathrm{Kpc})$ is also visible, and we can guess that there is another density peak around $6 \mathrm{Kpc}$. We also notice a clump in the histogram around a distance modulus of 17 (25 $\mathrm{Kpc}$ ), that is probably associated with the Sagittarius dwarf Galaxy. These results are completely new, and could be the starting point for some new modeling of microlensing towards the bulge.

\section{Acknowledgements}

It is a great pleasure to thank B. Paczyński for valuable suggestions and discussions. I would also like to thank G. Gilmore and L. Blitz for helpful discussions.

\section{References}

Alard, C., 1995, in preparation

Alard, C. et al., 1995a, A\&A, in press

Alard, C. et al., 1995b, in preparation

Paczyński, B. et al., 1994, AJ, 107, 2060

Udalski, A. et al., 1994, Acta Astron, 44, 165

Szymański, M. \& Udalski, A., 1995, private communication

Van Der Hooft, F., 1995, private communication

Weinberg, M., 1992, ApJ, 384, 81 